\title{
Image Reconstruction Using Wavelet Method
}

\author{
Dr. Nagabhushana
}

Department of Computer Science and Engineering, SJMIT Chitradurga, India-577501

Email: hod.cse@sjmit.ac.in

Dr. Aravinda T V

Department of Computer Science and Engineering, SJMIT Chitradurga, India-577501

Email: arvind_cta@yahoo.co.in

A V Radhika

$4^{\text {th }}$ Semester, M.Tech. Department of Computer Science and Engineering, SJMIT Chitradurga, India-577501

Email: radhilec18@gmail.com

ABSTRACT-

This paper proposes a joint system wherein lifting-based, divisible, image matched wavelets are assessed from compressively detected pictures and are utilized for the recreation of the same. Matched wavelet can be effectively composed if full picture is available. Additionally contrasted with standard wavelets as sparsifying bases, coordinated wavelet may give better recreation brings about compressive sensing application. Since in application, we have compressively detected pictures rather than full picture. Existing strategies for outlining coordinated wavelets can't be utilized. Accordingly, we propose a joint structure that appraisals coordinated wavelets from compressively detected pictures and furthermore recreates full pictures. This paper has three huge commitments. To begin with, lifting-based, picture coordinated distinct wavelet is planned from compressively detected pictures and is additionally used to recreate the same. Second, a straightforward detecting network is utilized to test information at sub-Nyquist rate with the end goal that detecting and reproduction time is diminished extensively. Third, another multi-level L-Pyramid wavelet disintegration system is accommodated divisible wavelet usage on pictures that prompts enhanced reproduction execution. Contrasted with CS-based remaking utilizing standard wavelets with Gaussian detecting lattice and with existing wavelet decomposition system, the proposed strategy gives speedier and better picture reproduction in compressive sensing application.

Keywords - Pattern recognition, Matched wavelet, multi-level L-pyramid wavelet decomposition, Nyquistrate, Gaussian sensing matrix, Image reconstruction, PCIM- partial canonical identity matrix, PCI - partial canonical identity.

\section{INTRODUCTION}

Classical signal acquisition technique in signal processing branch involves sensing the full signal at or above the Nyquist rate. In normal, this signal is mutated into a domain where it is compressible. Only some of the considerable coefficients of transformed signal having ample amount of energy are gathered and transmitted to the receiver along with the position intelligence to the transmitted coefficients. To recover the original signal, receivers are used to decode the transmitted signal. Thus, this process covers sensing the full signal, despite most of the samples in the transformed regions are to be discarded

\section{EXISTING SYSTEM}

Motivated with the concept of transform learning and the utility of rational wavelet transform in audio and speech processing, this paper proposes Rational Wavelet Transform Learning in Statistical sense (RWLS) for natural images. The proposed RWLS design is carried out via lifting framework and is shown to have a closed form solution. The efficacy of the learned transform is demonstrated in the application of compressed sensing (CS) based reconstruction. The learned RWLS is observed to perform better than the existing standard dyadic wavelet transforms.

\section{Disadvantages of the existing system}

The disadvantages of the existing system are as follows:

- Images of huge sizes poses problems with storage and computation

- Design of imaging system with larger space bandwidth product is difficult

- It faces difficult calibration requirements.

\section{PROPOSED SYSTEM}

This paper addresses this problem from the point of view of image reconstruction. Below are the salient contributions of this paper:

- We propose design of an image-matched separable wavelet in the lifting framework from a compressively sensed image, which is also used to reconstruct the image.

- In general, Gaussian or Bernoulli measurement matrices are used in compressive sensing application. We propose to use partial canonical identity matrix (PCIM) to sample data at the sub-Nyquist rate such that sensing time is reduced considerably.

- For the separable 2D wavelet transform, a new multilevel wavelet decomposition strategy is proposed that leads to improved reconstruction performance. 
This paper proposes to use partial canonical identity (PCI) matrix to sub-sample images at the sub-Nyquist rate. We show results on time complexity and performance of the conventionally used sensing matrices and PCI sensing matrix to establish the use of latter in this work. New multi-level wavelet decomposition strategy hereby named as LPyramid wavelet decomposition, for separable wavelet on images and compares the performance with the existing wavelet decomposition strategy.

\section{Advantages of the proposed system}

The advantages of the proposed system are as follows:

- Reconstruction quality of the image sensed partially with PCI sensing matrix is good.

- In this method there is no unique basis unlike Fourier transforms of the existing method.

- Better reconstruction performance is observed with the proposed methodology compared to the existing methodology.

\section{Objectives of the proposed method}

The main objective of the proposed system is to provide faster and better image reconstruction in compressive sensing application using matched wavelets.

\section{Applications of the proposed method}

The applications of the proposed system are:

- The method is applied to time frequency analysis of ultrasonic echo waveform obtained by an ultrasonic pulse echo technique.

- Electronic power and control of rotating machines and of other electric machines is investigated by this method.

- Signal processing and control is widely used in chemical industry therefore this method appears naturally as a useful tool.

- Faster and better image reconstruction in compressive sensing application using this method.

\section{IMPLEMENTATION}

Module Description

The modules are:

1. Gaussian sensing matrix R-pyramid wavelet

2. Pci L-pyramid 5/3 Wavelet

3. Pci L-pyramid Matched Wavelet Design

\section{Gaussian sensing matrix R-pyramid wavelet}

Compressed sensing based reconstruction with Gaussian and Bernoulli sensing matrices is implemented using block compressed sensing [11]. This is to note that reconstruction with $\mathrm{PCI}$ sensing matrix requires only the position information of the sampled pixels instead of the information of all entries of $\mathrm{M} \mathrm{N}$ sensing matrix that simplifies reconstruction with PCI sensing matrix. We compare reconstruction results on three images: 'Beads', 'Lena', and 'House' respectively. The size of each image is 512512. We have chosen these images because they exhibit different spectral properties. For example, 'Beads' is rich in high frequencies; 'Lena' contains both low and high frequency contents, while 'House' is rich in low frequencies.

\section{Pci L-pyramid 5/3 Wavelet}

As the image changes from being rich in high frequency ('Beads') to mid-frequency ('Lena') to low frequency ('House') content, better and better reconstruction performance is observed with the proposed methodology compared to the existing methodology. This is owing to the fact that the proposed methodology works best for signals rich in low frequency contents. The quality of images reconstructed with the proposed methodology is better in terms of PSNR than the coarse estimate. This is to note that the difference between the coarse estimate and the proposed solution is only in using standard bior $5 / 3$ wavelet versus matched wavelet, while keeping the sensing matrix and wavelet decomposition strategy as same. This further establishes the significance of the matched wavelet vis-a-vis standard bior $5 / 3$ wavelet in the proposed work. At higher sampling ratio of $90 \%$, performance gain with the proposed methodology over the existing methodology is $3 \mathrm{~dB}$ with 'Beads', 4 $\mathrm{dB}$ with 'Lena', and $13 \mathrm{~dB}$ with 'House'. Since at higher sampling ratios, most of the input image samples are available upfront, hence, matched wavelet design is optimum. This provides very good performance and huge improvement over the existing methodology, particularly, for images rich in low frequency content. At lower sampling ratio of $10 \%$, performance gain with the proposed methodology over the existing methodology is $6.5 \mathrm{~dB}$ with 'Beads', $9 \mathrm{~dB}$ with 'Lena', and $11 \mathrm{~dB}$ with 'House'. In fact, standard methodology with standard wavelets almost fails in reconstructing images with any good quality at very low sampling ratios, while the proposed methodology still performs good.

\section{Pci L-pyramid Matched Wavelet Design}

With the serpentine scanning discussed above, we convert a given image into two 1-D signals: one with column-wise scanning and another with row-wise scanning. Henceforth, in the present Section, we present matched wavelet design methodology using compressively sensed 1-D signal. We will use these designs in the next Section to note the performance over images as separable wavelet transforms. The proposed methodology has three stages. In stage-1, we obtain coarse image estimate from compressively (partially) sensed samples using a standard wavelet. We call this a coarser estimate because the wavelet used is not matched to the given signal and hence, the original signal may not be that sparse over this wavelet compared to that with the matched wavelet. This will impact the reconstruction performance. In stage-2, we estimate matched analysis wavelet filter that provides sparser sub band wavelet (detail) coefficients than those obtained from the standard wavelet in stage-1. Using these estimated filters and the coarser signal estimate of stage- 1, we design all filters of the matched wavelet system. In stage- 3, we reconstruct signal from 
measured sub- samples using the matched wavelet estimated in stage- 2 .

In the application of CS-based image reconstruction, the proposed methodology of this paper, has three contributions:

1) Proposed use of PCI sensing matrix: that is computationally inexpensive compared to the existing Gaussian matrix but provides approx. 2-5 dB lower Performance compared to the existing Gaussian matrix

2) Proposed L-Pyramid wavelet decomposition that provides better results in CS-based image reconstruction compared to the existing R-Pyramid wavelet decomposition

3) Design of image-matched wavelets: wherein a wavelet[8] is designed from a compressively sensed image and is used for the reconstruction of the same. Hence, an image is recovered by employing a wavelet matched to it.

\section{EXPERIMENTS AND RESULTS}

In this section, we present CS-based reconstruction results

of images using image-matched wavelets[8] designed from compressively sensed images. We apply the proposed method on three natural images shown in the below figure. Images with different spectral contents have been selected. For example, House is rich in low frequencies, Beads is rich in high frequencies.

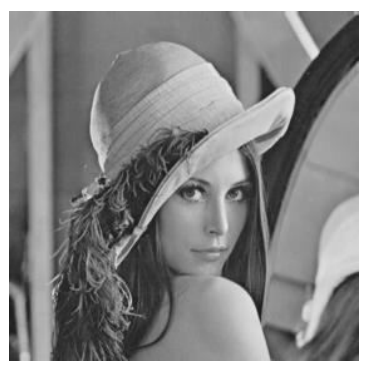

a) Lena

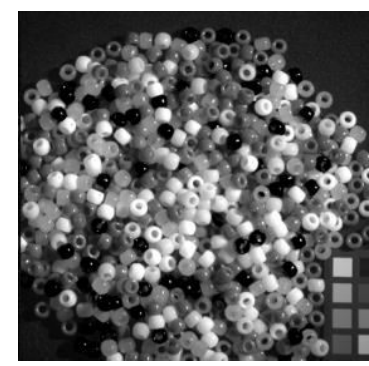

b)Beads

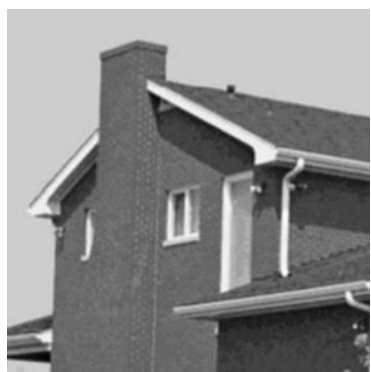

c)House

Fig. 1: Images used in experiments

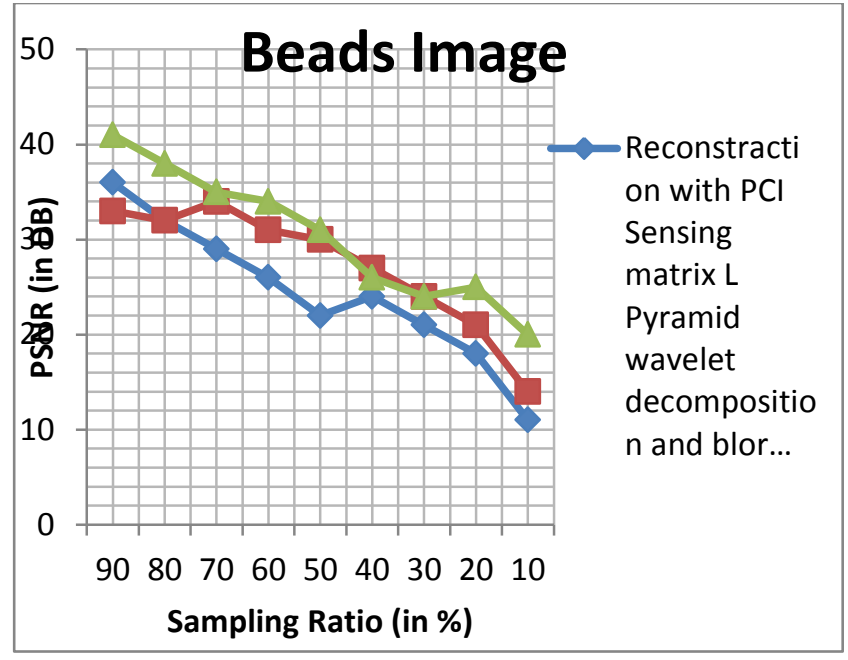

Fig. 2: CS-based reconstruction comparison of 1) the existing methodology: Gaussian sensing matrix, RPyramid wavelet decomposition, and $\mathrm{db} 4$ wavelet; 2) coarse estimate: PCI sensing matrix, L-Pyramid wavelet decomposition, and bior 5/3 wavelet; and 3) our proposed methodology: PCI sensing matrix, L-Pyramid wavelet decomposition, and proposed matched-wavelet design.

\section{SCREENSHOTS OF THE RESULTS}

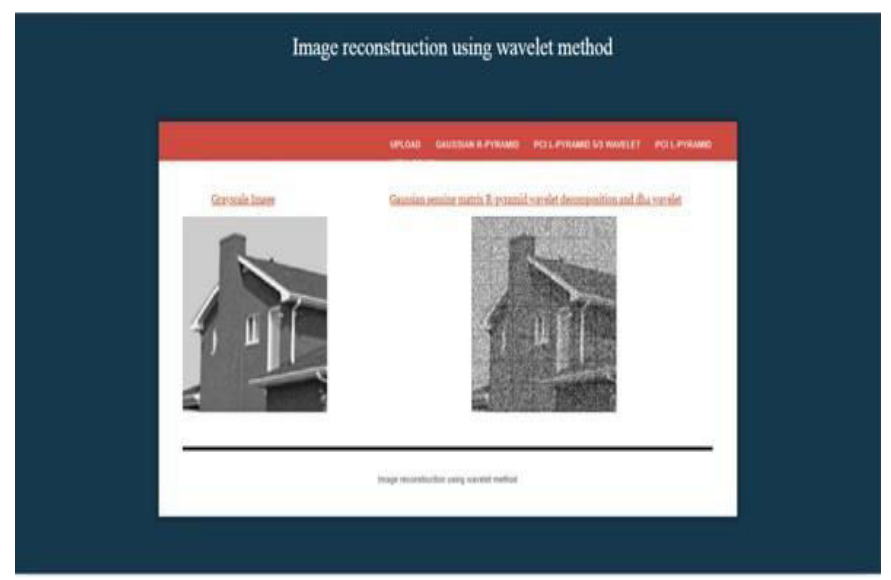

Fig. 3: The above figure shows the grayscale image and Gaussian sensing matrix R-pyramid wavelet decomposition and db-wavelet.

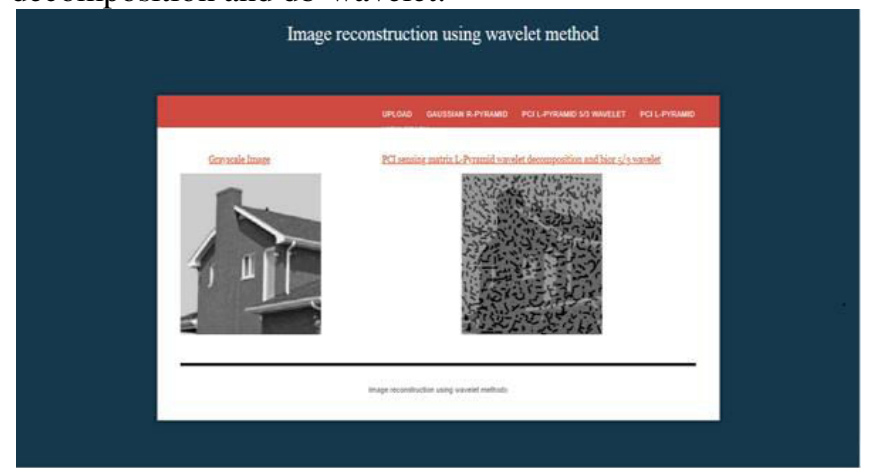

Fig. 4: The above figure shows PCI sensing matrix Lpyramid wavelet decomposition and bior $5 / 3$ wavelet 


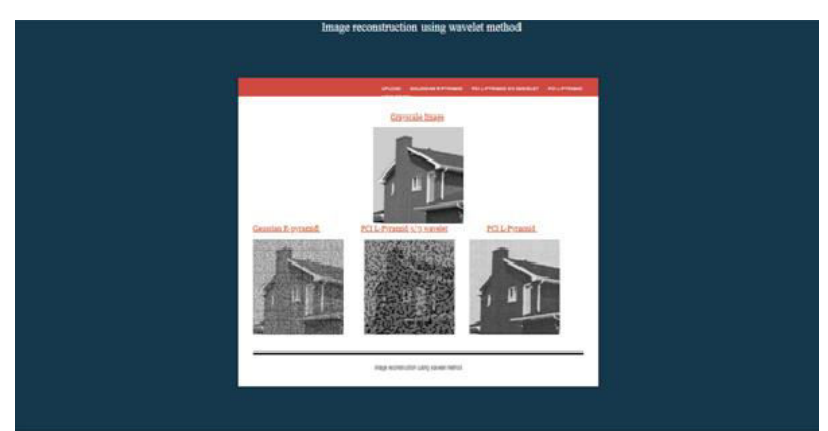

Fig. 5: The above figure shows the screenshot for all the three methods: Gaussian R-pyramid, PCI L-pyramid 5/3 wavelet and PCI L-pyramid

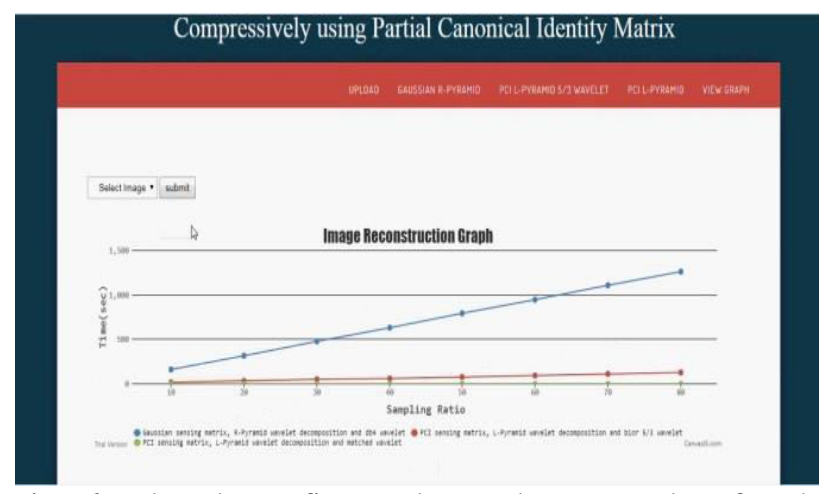

Fig. 6: The above figure shows the screenshot for the Image Reconstruction Graph

\section{CONCLUSION}

In this paper, we have proposed a joint framework wherein image-matched wavelets have been designed from compressively sensed images and later, used for reconstruction or recovery of the full image. We have also proposed to use a partial canonical identity sensing matrix for CS-based reconstruction of images that performs much faster compared to the existing Gaussian or Bernoulli matrices and hence, is suited for time-bound real-time reconstruction based applications. Although there is a slight degradation in performance with the proposed sensing matrix but that is easily covered up by the matched wavelet design. We have also provided a new multi-level L-Pyramid wavelet decomposition strategy that works much more efficiently compared to the standard wavelet decomposition method. Overall, the proposed work with different sensing matrix, new wavelet decomposition strategy, and image-matched wavelets provide much better reconstruction results with ease of hardware implementation in CS-based image reconstruction compared to the existing methodology.

\section{REFERENCES}

[1] E. J. Candès, J. Romberg, T. Tao, "Robust uncertainty principles: Exact signal reconstruction from highly incomplete frequency information", IEEE Trans. Inf. Theory, vol. 52, no. 2, pp. 489-509, Feb. 2006.

[2] D. L. Donoho, "Compressed sensing", IEEE Trans. Inf. Theory, vol. 52, no. 4, pp. 1289-1306, Apr. 2006.
[3] A. Gupta, S. D. Joshi, S. Prasad, "A new method of estimating wavelet with desired features from a givensignal", Signal Process., vol. 85, no. 1, pp. 147-161, Jan. 2005.

[4] A. Gupta, S. D. Joshi, S. Prasad, "A new approach for estimation of statistically matched wavelet", IEEE Trans. Signal Process., vol. 53, no. 5, pp. 1778-1793, May 2005.

[5] N. Ansari, A. Gupta, "Signal-matched wavelet design via lifting using optimization techniques", Proc. IEEE Int. Conf. Digit. Signal Process. (DSP), pp. 863-867, Jul. 2015.

[6] J. O. Chapa, R. M. Rao, "Algorithms for designing wavelets to match a specified signal", IEEE Trans. Signal Process., vol. 48, no. 12, pp. 3395-3406, Dec. 2000

[7] R. L. Claypoole Jr, R. G. Baraniuk, and R. D. Nowak, "Adaptive wavelet transforms via lifting," in Acoustics, Speech and Signal Processing, 1998.

[8] Daubechies and W. Sweldens, "Factoring wavelet transforms into lifting steps," Journal of Fourier analysis and applications, vol. 4, no. 3,pp. 247-269, 1998.

\section{BIOGRAPHIES AND PHOTOGRAPHS}

\section{Prof. Nagabhushana}

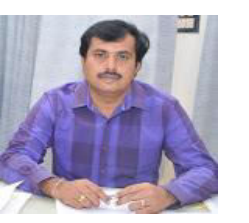

Prof. Nagabhushana is having around 14 years of experience in teaching field. Presently working as a professor and HOD in Computer science \& engineering department, SJM Institute of technology, Chitradurga. He has published more than 8 national and international papers. He completed his Ph.D in CSE in the year 2015 .

\section{Prof. Aravinda $T V$}

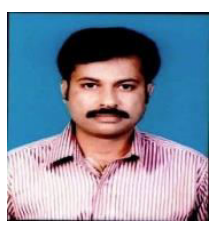

Prof. Aravinda T V is having around 14 years of experience in teaching field. Presently working as a professor in Computer science \& engineering department, SJM Institute of technology, Chitradurga. He has published more than 8 national and international papers. He completed his Ph.D in CSE in the year 2015.

\section{A V Radhika}

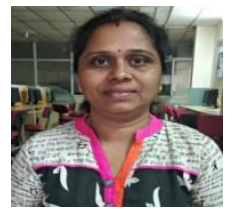

A V Radhika pursuing M.Tech.(Computer Science and Engineering) degree in Shri Jagadguru Mallikarjuna Murugarajendra Institute Of Technology, Chitradurga, India. Her area of interest encompasses Visual Cryptography, Digital Image Processing, and Web Applications Development. 